\title{
Reaching women in the Peruvian Andes through cervical cancer screening campaigns: assessing attitudes of stakeholders and patients
}

This article was published in the following Dove Press journal:

Patient Preference and Adherence

18 October 2016

Number of times this article has been viewed

\author{
John S Luque' \\ Jonathan N Maupin ${ }^{2}$ \\ Daron G Ferris ${ }^{3,4}$ \\ Wendy S Guevara \\ Condorhuaman ${ }^{4}$ \\ 'Department of Public Health \\ Sciences, Hollings Cancer Center, \\ Medical University of South Carolina, \\ Charleston, SC, ${ }^{2}$ School of Human \\ Evolution and Social Change, \\ Arizona State University, Tempe, \\ AZ, ${ }^{3}$ Department of Obstetrics and \\ Gynecology, Augusta University, \\ Augusta, GA, USA; ${ }^{4}$ CerviCusco, \\ Cusco, Peru
}

Background: Peru is characterized by high cervical cancer incidence and mortality rates. The country also experiences significant gaps in quality cervical cancer screening coverage for the population.

Objective: This descriptive mixed methods study conducted in Cusco, Peru, aimed to assess the attitudes and perceptions of medical staff, health care workers, and patients toward a cervical cancer screening program that included both clinic-based and community outreach services conducted by a nongovernmental organization clinic (CerviCusco). The study also explored patient knowledge and attitudes around cervical cancer and about the human papillomavirus (HPV) to inform patient education efforts.

Methods: The study employed structured interviews with key informants $(n=16)$ primarily from CerviCusco, which provides cervical cancer prevention, screening, diagnosis and treatment services, and surveys with a sample of patients $(n=30)$ receiving services at the clinic and at screening campaigns.

Results: The majority of key informant medical staff participants felt that the general public had a very negative view of government health services. One theme running throughout the interviews was the perception that the general population lacked a culture of preventive health care and would wait until symptoms were severe before seeking treatment. Regarding services that were received by patients at CerviCusco, the participants responded that the prices were reasonable and more affordable than some private clinics. Patients attending the rural health campaigns liked that the services were free and of good quality.

Conclusion: CerviCusco has demonstrated its capacity to provide screening outreach campaigns to populations who had not previously had access to liquid-based cytology services. The finding that patients had generally low levels of knowledge about cervical cancer and the HPV vaccine prompted the development of culturally and linguistically appropriate educational and promotional materials to improve the educational component of the periodic campaigns conducted primarily in rural areas of Andean Peru.

Keywords: Pap test, rapid assessment, global health, screening programs, outreach, South America

\section{Introduction}

Despite global advances in the prevention of cervical cancer with the introduction of the human papillomavirus (HPV) vaccine and attempts to deliver comprehensive cervical cancer screening programs, women from developing countries in Latin America continue to experience higher rates of cervical cancer morbidity and mortality than those in developed countries. Cervical cancer is the second most common cancer among women worldwide; however, it can be prevented when premalignant changes of the
Department of Public Health Sciences, Medical University of South Carolina, I 35 Cannon Street, Suite 303, MSC 835,

Charleston, SC 29425, USA

Tel + I 8438762248

Email luquej@musc.edu 
cervix are detected early and treated appropriately. Peru is characterized by high incidence of cervical cancer and mortality rates, with an age-standardized incidence rate of $32.7 / 100,000$ population and a mortality rate of 12.0/100,000 population. ${ }^{1}$ In comparison, according to GLOBOCAN 2012 , the overall mortality rate in South America is 8.6/100,000. In some other South American countries, the mortality rates are lower than that of Peru; for example, the mortality rate in Brazil is 7.3/100,000 population, Chile 6.0/100,000 population, and Colombia 8.0/100,000 population. ${ }^{2}$ In Peru, there were an estimated 4,635 new cervical cancer cases and 1,715 cervical cancer deaths in 2012, second only to stomach cancer in women; moreover, the disease was the leading cause of cancer deaths in women between 15 and 44 years of age. ${ }^{1}$

Early detection of cervical neoplasia with Pap tests is the optimal strategy to reduce the incidence and mortality rates in Peru, because in low resource settings, the cost of the HPV vaccine is too expensive for most Peruvians. ${ }^{3}$ Because of the relatively high costs of the Pap test and variable quality of samples, several prevention campaigns in Peru have employed the lower-cost, visual inspection with acetic acid (VIA) screening method followed by cryotherapy for cervical lesions. ${ }^{4,5}$ A successful Pap testing program requires an investment in training for cytotechnologists and cytopathologists, as well as capacity building for essential supplies and quality laboratory equipment. Moreover, because regular cervical cancer screening using Pap tests requires resources and a quality control program for both screening and follow-up, capacity for delivering cancer education, and regular contact with health care providers who recommend screening, low-resource countries face challenges in reaching a large proportion of population, especially in rural areas. ${ }^{6}$

Peru's cervical cancer screening guidelines are targeted at women between 30 and 50 years and include annual Pap tests, VIA every 2 years, and the HPV DNA test every 5 years. ${ }^{7}$ Rough estimates place screening coverage at $51 \%$ of the eligible population, but there are reported variations in rural $(42 \%)$ and urban $(57 \%)$ areas. ${ }^{1,8}$ One large study carried out in 20 of the most populous cities reported Pap test coverage to be $\sim 31 \%$ among young women (18-29 years old). ${ }^{3}$ The study found regional differences, with Andean women significantly less likely to be screened than women from the coast.

In low-resource settings such as Cusco, Peru, and the surrounding rural communities in the Peruvian Andes, structural barriers to cervical cancer screening include lack of access to health care, shortage of quality facilities and laboratories (outside of Lima, few facilities can perform cone biopsies or colposcopies), few trained personnel, distance to health care facilities, irregular hours of operation, long waiting times, and lack of affordable options for follow-up care. ${ }^{9}$ Individual and cultural barriers include lack of preventive health-seeking behaviors, low exposure to biomedical procedures, poor knowledge of cervical cancer, language barriers (for nonSpanish speakers), negative views of health care providers, lack of spousal support for health care seeking, anxiety and fear over potential positive cancer diagnoses, shortage of female providers, and modesty regarding exposure of one's body. ${ }^{3,5,10}$ Facilitators to screening include peace of mind after receiving negative results, sense of control and empowerment over one's own health, support from friends and family, and reassurance against the detection of other sexually transmitted infections or vaginal infections. ${ }^{10}$

In terms of HPV vaccination, there was a demonstration project conducted by the Peru Ministry of Health (MINSA) in 2008 for school-based vaccination to girls in the 5th grade in three areas of the country, but not in Cusco, the setting for this study. A qualitative study of decision-making processes among parents in Piura in northwestern Peru found that most parents accepted the new vaccine, but some parents expressed an overall lack of confidence in the health system, which reduced their enthusiasm. ${ }^{11,12}$ HPV vaccination coverage rates for Peru are only available as estimates from demonstration projects in circumscribed areas; however, the 2014 estimate for the full course of HPV vaccination for girls between 10 and 14 years in South America is $40 \%{ }^{13}$ This overall percentage overestimates coverage in Peru since there have been stronger HPV vaccination programs in other South American countries, like Argentina, where rates for full course coverage have been estimated at $52 \% .{ }^{14}$

\section{Setting}

CerviCusco, a Peruvian registered nongovernmental organization (NGO) located in Cusco, is committed to improving the health and quality of life of Peruvian women through the primary and secondary prevention of cervical cancer. ${ }^{15}$ In addition to providing low-cost, clinic-based services since 2008, CerviCusco also conducts free medical outreach campaigns to provide access to care for those women who are unable to travel to Cusco. CerviCusco collaborates with MINSA, academia, private health care systems, local health care providers, medical device companies, and the community to foster community acceptance and ensure program sustainability for these campaigns. CerviCusco is the only health facility in the mountain region of Peru to offer liquid-based cytology, wherein samples are transferred to vials for analysis. 
CerviCusco currently provides cervical cancer screening to over 10,000 women annually. It screened 9,998 patients in 2014 and 13,397 patients in 2015 with the liquid-based Pap test. Of the patients screened in 2014, 1,386 (14\%) patients had abnormal results and 444 received further diagnosis using colposcopy. In 2014, 134 Cono loop electrosurgical excision procedure (LEEP) procedures were conducted, and there were 9 confirmed cases of invasive cervical cancer. In 2015, there were 11 confirmed cervical cancer cases.

This descriptive, mixed method case study conducted in Cusco, Peru, aimed to assess the attitudes and perceptions of medical staff (doctors, nurses, and nurse midwives), health care workers, and patients toward both clinical services and outreach screening campaigns delivered by CerviCusco. The purpose of conducting these key informant interviews was to characterize these attitudes and perceptions to provide feedback to CerviCusco leadership in order to improve quality and monitor dissemination of evidence-based clinical practice. This study primarily interviewed key informants who either worked directly for CerviCusco or worked for the MINSA and collaborated with CerviCusco to gather in-depth, confidential opinions. The study also explored knowledge, attitudes, and beliefs of cervical cancer of CerviCusco patients and collected information about their opinions of CerviCusco services. The results of the study findings contributed to the improvement of the quality of services and prompted the development of culturally and linguistically appropriate educational and promotional materials to improve the educational component of the outreach campaigns.

\section{Methods}

This study employed qualitative interviews with key informants and surveys with patients. All research participants completed a written informed consent process prior to being interviewed or surveyed. The study was approved by the institutional review boards of the National Institute of Health of the Peruvian Government (INS), Augusta University, and Georgia Southern University.

\section{Medical staff informant interviews}

In spring 2014, the study team employed a structured instrument (interview guide composed of 12 open-ended questions) to interview the medical staff and health care workers in Cusco and health care workers and government officials in a small rural community (district of Quiquijana in Quispicanchi Province) where a recent outreach campaign had taken place. All medical staff and health care workers who were interviewed were either employed by CerviCusco or worked as nurse-midwives or doctors in the health department and were delivering screening samples to be analyzed. The purpose of the interviews was to collect information on major health problems in the Cusco region; community attitudes toward health, prevention, and health services; community and health care personnel attitudes toward CerviCusco services; and identify recommendations to address any problems identified with CerviCusco clinical operations. Participants included eight medical staff and health care workers from CerviCusco (which included nurses, nurse-midwives, administrative staff, a doctor, and a laboratory cytotechnologist), two from the Quiquijana government social welfare office, and six nursemidwives or doctors from MINSA in Cusco (including 1 from Quiquijana). The interviews were conducted in Spanish, recorded and transcribed, and analyzed by question for themes using MAXQDA 11 (Marburg, Germany). Quotes and response summaries illustrate key themes.

\section{Patient survey}

To explore patient data related to cervical cancer, surveys were administered to CerviCusco patients incorporating both open-ended and close-ended questions after they received a cervical cancer screening examination either in CerviCusco or at an outreach campaign. The open-ended questions covered topics including community health resources and attitudes toward CerviCusco services; beliefs and attitudes about common health conditions, prevention, causes, and treatments; patients' health history; and cancer knowledge. For close-ended questions, participants answered questions about their knowledge and beliefs about HPV/cervical cancer drawn from the Spanish language version of the National Cancer Institute's Health Information National Trends Survey. ${ }^{16}$ Participants also responded to agree/disagree questions on attitudes and beliefs toward cervical cancer that had been used in a previous survey with Latina immigrant women in the US. ${ }^{17}$ In addition, patients reported demographic information including age, years of formal education, marital status, weekly income, family size, and language acculturation measured on a 4-point language acculturation scale. ${ }^{18}$

From March to June 2014, the study team administered 30 surveys to a convenience sample of women aged 30-49years who had just received a Pap test from CerviCusco. Surveys took $\sim 45$ min to administer. Ten surveys were completed in the CerviCusco clinic, and 20 surveys were completed during screening outreach campaigns in four rural areas where campaigns were being held (Chinchaypucyo, Anta Province; Calca, Calca Province; Maras, Urubamba Province; and Urcos, Quispicanchi Province). Descriptive statistics 
(means/ranges and numbers/percentages) were calculated. Open-ended questions were summarized for most frequent responses and then categorized.

\section{Results}

\section{Key informant interviews - medical staff and health care workers}

\section{Participant demographics}

Of the 16 participants, 14 were female and 2 were male. Half of the participants worked directly for CerviCusco, and the other half of the participants were collaborators with CerviCusco but worked for government agencies. Key informant demographic characteristics are summarized in Table 1.

\section{CerviCusco procedures for screening and follow-up} CerviCusco medical staff reported that any patients with atypical results (eg, ASCUS, LSIL, and HSIL) were called for follow-up and received a colposcopy examination if necessary for further diagnosis. Patients with abnormal results attending outreach campaigns were told to return to their local MINSA office after 1 month to receive their results. In the case of abnormal results, they were scheduled for follow-up at CerviCusco. The clinic also performed follow-up diagnostics with biopsies. With the more serious cases requiring radiotherapy and chemotherapy, patients were usually referred to the Instituto Nacional de Enfermedades Neoplásticas (National Cancer Institute) in Lima. Cone biopsies or LEEP could be done in CerviCusco only when

Table I Demographic characteristics of interview participants

\begin{tabular}{ll}
\hline Characteristic & Total (N=16) \\
\hline Age, years & $37(27-63)$ \\
Years of schooling & $18(16-27)$ \\
Average income/month (soles) & $4,877^{*}$ \\
Occupation & \\
Nurse-midwife & $6(37 \%)$ \\
Nurse & $2(13 \%)$ \\
Doctor & $3(19 \%)$ \\
Administrative or other & $5(31 \%)$ \\
Affiliation & \\
CerviCusco & $8(50 \%)$ \\
MINSA or other government agency & $8(50 \%)$ \\
Marital status & \\
Married/living with a partner & $9(56 \%)$ \\
Single/other & $7(44 \%)$ \\
Residence & \\
Rent & $4(25 \%)$ \\
Own & $5(31 \%)$ \\
Other & $7(44 \%)$ \\
\hline
\end{tabular}

Notes: Data are presented as mean values (ranges) for continuous variables and frequencies (percentages) for categorical variables. *I Peruvian Nuevo Sol $=0.30$ USD. Abbreviation: MINSA, Peru Ministry of Health. the visiting physician was present, which was approximately during a 1-week period every other month. Because of the poor economic situation of many patients, seeking treatment in distant Lima was not a realistic option.

\section{Personal experiences with cervical cancer patients}

Participants were asked to share brief vignettes about patients they knew who had cancer. The themes throughout these stories emphasized late diagnoses and financial constraints to secure treatment. Because of the high rates of cervical cancer in Peru, one doctor reported that many unnecessary cone biopsies were being done on healthy women in their 20 s at one of the regional hospitals. However, there were some rare cases discussed of women in their 20s and 30s who had advanced cervical cancer. Other patients were able to receive simple hysterectomies in Cusco at a public or regional hospital.

\section{Perceived community attitudes toward health care}

The majority of medical staff participants felt that the general public had a very negative view of government health services. They attributed these perceptions to patient complaints of: 1) long wait times; 2 ) difficulty in getting referrals to a specialist; 3 ) not receiving test results in a timely manner (or ever) - up to 6 months for Pap test results was reported by one doctor; 4) lack of caring attitudes from health care workers; 5) having to pay more for a private clinic because of inefficiencies in the government system; and 6) having to fill out a lot of paperwork for free or low-cost services. These issues were also recognized by medical staff and government officials themselves. One Quiquijana government official stated that medical services were suboptimal because of "tight government budgets, poor remuneration of health care personnel, and a shortage of medicines." There was reportedly poor infrastructure and maintenance of facilities in the region. One doctor explained that in the Social Security hospital, oftentimes women would have trouble scheduling their appointments because "there are very few gynecologists in Cusco" or they would miss their appointments because of "other priorities." She indicated that in her private practice, many women in their 40s and 50s had never had a Pap test, thus explaining the high rates of cervical cancer. This doctor noted cultural factors and commented on a common conversation with her patients,

Why don't you go to the health center to get a Pap test?

But the Quechua speaking patient, patient in the rural area, doesn't like her genitals to be touched.

One theme running throughout the interviews was the perception that the general population lacked a culture of 
preventive health care and would "wait until symptoms were severe before seeking treatment." Participants stated that many patients had little education about their health. For example, one nurse-midwife in a rural community stated,

there are many cases of cancer in women, because there is little prevention, and there is a lack of knowledge about the importance of the Pap test.

Another nurse-midwife who worked in a mobile unit explained that she was constantly "fighting" with women to have them get their Pap. The CerviCusco staff indicated that Quechua women showed the most resistance and that they were not aware of the risk factors or symptoms of cervical cancer. According to the medical staff, Quechua women held many ethnomedical beliefs about the earth, that one can contract illnesses from the wind, and with a "cleansing" from a curandero, or natural healer, one would get better. In addition, cultural attitudes such as machismo and problems like alcoholism, which contributed to domestic abuse, were issues that impeded medical care for women. Reportedly, many women of low education backgrounds did not want to see a male doctor for gynecological examinations, because of past experiences of spousal abuse and controlling husbands. One male nurse-midwife reported that he did not feel his gender affected his ability to perform Pap tests; however, there was a language barrier because he did not speak Quechua and the patients were very reserved about exposing their genitalia. Finally, key informants indicated that some people had low access to health care because of geographical barriers (rurality).

\section{Attitudes toward CerviCusco services}

Overall, all the health care worker participants indicated their patients were very much satisfied with the services they received at CerviCusco. A nurse-midwife from the public sector remembered that before CerviCusco, Pap tests were sent to one of two hospitals, but now the tests were sent directly to CerviCusco. He was pleased that results were received much faster now, that is, in 10 days. He indicated that the view of his practice had improved because the services were free or low cost.

The main problem CerviCusco staff reported was variability in turnaround for liquid Pap test results. Sometimes results were timely in 2-3 days, but occasionally they could be delayed for a few weeks because of an excessive workload. To receive their results, patients would either have to call or return to CerviCusco. For women who received Pap tests in the campaigns, they would have to return to the health department that collaborated with CerviCusco to receive their results after 1 month. In Quiquijana, the women did not receive written results but only received the results verbally from the nurse-midwife who maintained the patient charts.

The majority of the CerviCusco staff suggested that the clinic could improve patient volume by having a site or a satellite office more centrally located in Cusco, having only morning operating hours like other clinics - which has now been implemented - and improving the marketing of the clinic rather than just relying on word-of-mouth. One of the doctors collaborating with CerviCusco also suggested the need for more equipment and services at CerviCusco, such as urinary and blood laboratory examination equipment, X-ray, cobalt therapy, and a pharmacy. None of these facilities were in the same neighborhood as CerviCusco, which is located about $20 \mathrm{~min}$ from the city center, thus presenting patients with additional follow-up challenges to address their health conditions depending on where they lived. For cancer patients, the problem was that there were no radiotherapy services in Cusco, and patients would have to wait for 2-3 months to receive these services in Lima or Arequipa.

\section{Patient survey}

\section{Participant demographics}

Table 2 describes the demographics of the 30 survey participants. The average age of the participants was 40 years, with an average of 6.5 years of formal schooling. The women

Table 2 Demographic characteristics of survey participants

\begin{tabular}{ll}
\hline Characteristic & Total $(\mathbf{N}=\mathbf{3 0})$ \\
\hline Age, years & $40(30-49)$ \\
Years of schooling & $6.5(0-18)$ \\
Number of people in household & $4(2-7)$ \\
Median income category/week (soles) & $201-400 *$ \\
Score on language acculturation scale & $0.7(0-4)$ \\
(0= low to 4= high) & \\
Self-identified ethnic group & \\
Mestizo & $15(50 \%)$ \\
Quechua & $15(50 \%)$ \\
Marital status & \\
Married/living with a partner & $16(87 \%)$ \\
Single/other & $4(13 \%)$ \\
Currently employed & $20(67 \%)$ \\
Regular health care provider & $9(30 \%)$ \\
Health insurance & $24(80 \%)$ \\
Residence & \\
Rent & $21(70 \%)$ \\
Own & $6(20 \%)$ \\
Other & $3(10 \%)$ \\
\hline
\end{tabular}

Notes: *I Peruvian Nuevo Sol $=0.30$ USD. Data are presented as mean values (ranges) for continuous variables, and frequencies (percentages) for categorical variables. a Modified version of the Acculturation Scale for Mexican Americans was used to measure acculturation (substituting "Quechua" for "English" language). ${ }^{18}$ 
were mostly Quechua-dominant speakers $(21 ; 70 \%)$ based on the low acculturation score for Spanish language $(0.7)$ on the 4-point language acculturation scale. The median weekly income range was 201-400 soles (74-148 USD).

\section{Community health resources and attitudes toward the campaigns}

The first part of the survey included open-ended questions about health topics. When asked about health resources in the community, participants most frequently mentioned health centers and curanderos. Other resources listed included nurse-midwives, private clinics, and regional hospitals. Participants were asked to comment on the services they received at CerviCusco. The clinic patients had to pay for services, unlike those who received free services at the CerviCusco outreach campaigns. The clinic patients said that the prices were reasonable and more affordable than the private clinics. Patients attending the campaigns liked that the services were free and of good quality. Critical comments included having to wait in queue, not being able to communicate in Quechua with health care staff, lack of Spanish skills among the American doctors, and not having additional free health services, such as physical examination or blood sugar testing. CerviCusco has two nurse-midwives who are bilingual in Quechua and Spanish, and the Spanish-speaking CerviCusco doctor speaks basic Quechua. Sometimes health care professional volunteers from the US would assist with the outreach campaigns, so patients would require translators. Since the surveys were taken at the time of screening, some participants expressed the hope that the results would arrive promptly, since results could potentially be delayed based on their past experiences with health care.

\section{General health conditions and health services}

Participants responded to a question about where they normally received health services. Most women answered that they received services at the health department (centro or puesto de salud). Two participants indicated that they would first use home remedies or a curandero before going to the health center. The type of services they received at a health center or a private clinic included medical, dental, nursing, obstetrics, lab, colposcopy, injections, family planning, psychology, and pediatrics. Only nine (30\%) women reported that they had a regular health care provider, such as a doctor at the health department or a private clinic.

\section{Prevention and cervical cancer knowledge}

When asked what tactic one can use to prevent disease, the most common response was to wear warm clothes and drink hot tea. Other preventive measures that they mentioned included not taking antibiotics, personal hygiene, avoiding fast food and street vendors, eating fruits and vegetables, taking vitamins, and getting regular check-ups. Several women mentioned good hygiene, receiving regular medical checks, and limiting the number of sexual partners as methods to prevent cancer. Regarding prevention of cervical cancer, women most commonly listed getting a yearly Pap test. Other methods included limiting number of sexual partners, washing private areas with herbs, not having an abortion, and good hygiene.

Nine $(30 \%)$ participants said they had never heard of cervical cancer. Of those who had heard of it, most said they had heard about it in the health department, but some also said they had heard of it from a friend or in the media. Most participants answered that they did not know what treatments existed for cancer; however, some responses included, "it is incurable, there is no treatment," and "taking different medicines." Some participants also responded, "there is no cure when it is advanced," "if a woman doesn't have money to get treatment, she will die," and "when someone has cancer, it is not possible to get better." Participants were also asked whether they knew anyone who had ever told them that she had cervical cancer. Eight (27\%) women reported that they had a friend or someone they knew who had told them they had cervical cancer.

\section{Pap test history and HPV knowledge}

Since the patient surveys were administered after women had just been screened, all participants surveyed had recently received a Pap test. Twenty-four (80\%) women reported that a health care provider had recommended a Pap test. Most women reported receiving their Pap tests in the campaigns or at the health department. Table 3 summarizes the participants' health history, cervical cancer screening, and HPV knowledge. When asked how long before their most recent Pap test did they receive the previous one, 10 (33\%) women reported in the last year, $8(27 \%)$ in the last 2 years, $5(17 \%)$ in the last 3 years, $3(10 \%)$ women over 3 years ago, and the rest (13\%) had never received a Pap test before. The female participants were asked whether they had any concerns about the Pap test. Those who expressed concerns listed a number of reasons including: fearing that tubal ligation could cause cancer or an infection, having vaginal secretions, and fearing that ulcers that could be cancer. Women were asked how often they should have a Pap test. Sixteen (53\%) participants reported 1 year or less, $5(17 \%)$ participants listed between 1 and 3 years, and the rest (9\%) did not know. 
Table 3 Health history, cervical cancer screening history, HPV knowledge, and cervical cancer attitudes and beliefs among survey participants

\begin{tabular}{|c|c|}
\hline Survey item & Total $(\mathbf{N}=\mathbf{3 0})$ \\
\hline $\begin{array}{l}\text { Pap test before the most recent Pap test was } \\
\text { within the past } 3 \text { years }\end{array}$ & $23(77 \%)$ \\
\hline Expect to have another Pap test in the next year & $21(70 \%)$ \\
\hline $\begin{array}{l}\text { Had received a provider's recommendation for } \\
\text { a Pap test }\end{array}$ & $24(80 \%)$ \\
\hline Had received family planning services & $22(73 \%)$ \\
\hline Had heard of HPV & $6(20 \%)$ \\
\hline Thought HPV caused cervical cancer & $6(20 \%)$ \\
\hline Thought HPV was sexually transmitted & $8(27 \%)$ \\
\hline Thought HPV was common & $4(13 \%)$ \\
\hline $\begin{array}{l}\text { Thought HPV would go away on its own } \\
\text { without treatment }\end{array}$ & $5(17 \%)$ \\
\hline Thought HPV could cause abnormal Pap tests & $5(17 \%)$ \\
\hline Had heard of the HPV vaccine & $5(17 \%)$ \\
\hline $\begin{array}{l}\text { Thought the HPV vaccine was an effective way } \\
\text { to prevent HPV infection }\end{array}$ & $2(7 \%)$ \\
\hline If had HPV, would tell their partner? & $22(73 \%)$ \\
\hline $\begin{array}{l}\text { Thought if cervical cancer is found early, it can } \\
\text { be cured }\end{array}$ & $26(87 \%)$ \\
\hline $\begin{array}{l}\text { I would undergo cervical cancer treatment that } \\
\text { is unpleasant or painful if it would increase my } \\
\text { chances of living longer }\end{array}$ & $30(100 \%)$ \\
\hline I would rather not know if I had cervical cancer & $19(63 \%)$ \\
\hline $\begin{array}{l}\text { I would be afraid to tell my husband or partner } \\
\text { if I had cervical cancer }\end{array}$ & II (37\%) \\
\hline $\begin{array}{l}\text { I need a Pap smear only when I experience } \\
\text { vaginal bleeding other than menstruation } \\
\text { (or when I experience other symptoms) }\end{array}$ & $16(53 \%)$ \\
\hline $\begin{array}{l}\text { There is not much I can do to prevent } \\
\text { cervical cancer }\end{array}$ & $15(50 \%)$ \\
\hline $\begin{array}{l}\text { I am very likely to get cervical cancer sometime } \\
\text { in my lifetime }\end{array}$ & $12(40 \%)$ \\
\hline
\end{tabular}

Note: Data are presented as frequencies (percentages) of participants who reported a characteristic or agreed with the statement.

The survey also included a number of questions about HPV and the HPV vaccine. Only 6 (20\%) women said they had heard of HPV. Of those who had heard of HPV, most said they had heard about it at the health department. Only 2 women had been told by a doctor that they had a HPV infection. Five (17\%) women had heard of the HPV vaccine. These participants listed that lack of knowledge, money, and fear of injection pain as potential reasons would keep someone from getting the vaccine. When asked what would motivate women to get the vaccine, responses included education, low cost or free product, promotion, and good treatment of patients. Participants were also asked to agree or disagree with attitudes and beliefs about cervical cancer and screening. All participants agreed with the statement, "I would undergo cervical cancer treatment that is unpleasant or painful if it would increase my chances of living longer," and 26 (87\%) participants agreed with the statement, "if cervical cancer is found early, it can be cured." Half of participants agreed that there was not much they could do to prevent cervical cancer, and $63 \%$ of the participants would rather not know if they actually had cervical cancer.

\section{Discussion}

In this study, both the key informants and the patients described individual and structural barriers to accessing cervical cancer screening services, follow-up, and treatment in the public health sector. Similar to a previous study, major problems identified with health services in Peru included lack of resources and difficulty in accessing cancer treatment services, since they were centralized in either Lima or Arequipa. ${ }^{5}$ However, the same participants reported that superior services and timely follow-up were available from private and NGO clinics. CerviCusco is unique in the Cusco region since it functions as a stationary clinic and also provides mobile clinic outreach services. Although there were some challenges identified by patients in the screening outreach campaigns, such as waiting in queues and not having additional health services besides the Pap test, such as screening for other health conditions, there was an overwhelmingly positive response to the services received and confidence in the professionalism of the CerviCusco medical staff.

The efforts of CerviCusco to provide screening opportunities through screening outreach campaigns addresses one of the primary barriers to women receiving timely screening (eg, poor access to health services). There is evidence from evaluations of other screening campaigns in Peru that both community promotional events and static screening facilities are needed to increase screening participation. ${ }^{4,19,20}$ The convenience of accessing screening opportunities in both urban and rural areas around Cusco allowed community participants to receive services, many for the first time. As a previous study in Peru recommended, a national campaign to educate the public and ensure regular screening and follow-up would be one effective means to increase screening coverage. ${ }^{5}$ Since Peru is similar to some other South American countries, like Colombia, Ecuador, and Bolivia, where cervical cancer screening is only available in certain regions, Peru would benefit from more cervical cancer screening outreach in other areas of Peru where screening is not easily accessible. ${ }^{21,22}$ To address structural barriers and screening access in Peru, different strategies have been employed to expand cervical cancer screening using low-cost methods such as VIA. Although there are inherent advantages to rapid diagnostic methods, such as VIA, these are offset by suboptimal diagnostic accuracy which can lead 
to inappropriate treatment for many and undetected disease in others. This tension between physician preferences for VIA versus conventional Pap test was identified in a survey of Bolivian providers who believed VIA would be preferable to women because of their cultural preference for wanting rapid results and not trusting results that would not be ready for several weeks. However, professionally, there was a national program advocating Pap testing; hence, a significant minority of providers favored Pap test over VIA. ${ }^{23}$

To address poor access to quality health care for the indigenous population, CerviCusco has evaluated a program of temporary screening clinics held in public marketplaces frequented by the target population on a weekly basis. ${ }^{24}$ Patients reported a preference for having their Pap test in market areas in tents since it was more convenient and accessible than going to the clinic. However, other bottlenecks in the system remain, such as cost and transportation barriers to receive follow-up treatment for abnormal Pap test findings. ${ }^{19,25}$ Hence, multiple deficiencies in the current cancer prevention program in Peru exist. For middle-income countries, many challenges remain for implementing highquality Pap test programs. ${ }^{26}$ With newer options becoming available even in poor-resource countries, a new cervical cancer prevention model based on limited interval HPV testing and widespread HPV vaccination may offer hope for reducing the high rates of these preventable cancers. ${ }^{20,27}$

The key informant interviews suggested that radio is the principal medium for advertising health screening events, and the patient surveys indicated that most people own a radio. Yet, advertising adds additional expense to health care resources that are already challenged financially. CerviCusco is currently working to expand its marketing and outreach efforts to boost patient volume in both the clinic and community setting by using social media and radio advertising. The clinic has also standardized their operating hours, and now they are open Monday through Saturday from 8 am to $2 \mathrm{pm}$. CerviCusco has experience with educating patients through culturally sensitive educational videos in the Quechua language to reduce anxiety about Pap and colposcopy examinations. ${ }^{28}$ An additional video describes the electrosurgery procedure in both Quechua and Spanish. ${ }^{29}$ Building on these previous successes, the study team developed Spanish-language photonovellas and Quechua-language radio advertisements as part of an educational outreach strategy to increase knowledge about cervical cancer. ${ }^{30}$ These materials are now being used to better inform patients about the purpose and benefits of cervical cancer screening. These efforts address the marketing deficiencies that were identified in the key informant interviews. Furthermore, CerviCusco hired a marketing professional to lead fundraising efforts and other promotional activities. In addition, a team from BD Pharmaceuticals and IBM, in partnership with Direct Relief, participated in volunteer service trips between 2014 and 2016. Their efforts helped to expand laboratory facilities, improve laboratory design and infrastructure, implement an electronic medical record system, improve marketing, and develop engineering solutions to streamline clinic operations. ${ }^{31}$

Individual barriers to screening in Peru include having other priorities and fear of cancer as a death sentence. ${ }^{10}$ In addition, low knowledge of HPV and cervical cancer makes the purpose of screening unclear. ${ }^{32}$ The low knowledge of cervical cancer/HPV and low education levels among our patient participants may be associated with their fatalistic beliefs expressed in their responses to the questions about their chances of getting cervical cancer and interest in knowing about a hypothetical cervical cancer diagnosis. The current study found that only $20 \%$ of patients thought HPV was linked to cervical cancer. This is lower than a larger survey study completed in Lima in 2006 which found that $38 \%$ knew of the link. ${ }^{33}$ While considering disease prevention, ethnomedical beliefs and reducing exposures are the predominant concerns. The patient knowledge data from this study are similar to other studies in Peru which have found that there is limited knowledge and consensus about cervical cancer causes, screening, and treatment options., The belief that cervical cancer was a deadly disease was reinforced by survey findings which found that half of participants "did not think there was anything they could do to prevent cervical cancer." In this study, screening was not necessarily viewed as a preventive practice, and there were negative consequences associated with screening. Lack of awareness of cervical cancer among this Quechua language dominant patient population could also be linked to low Spanish literacy since most posters and educational materials found in health departments are in Spanish.

This descriptive case study was an assessment of a particular nonprofit clinic; hence, the intent was not to generalize to other regions of Peru, but to assess one model for how a quality cervical cancer screening program might be instituted in a low resource country. The study only interviewed key informants who either worked for or collaborated directly with CerviCusco (except for the small sample from Quiquijana), thus some findings related to the quality of clinic services and patient satisfaction might have been positively biased. The survey was based on a small sample 
size; hence, inferential statistics could not be used to analyze the factors associated with health care utilization among patients. The intent of the survey was to offer a snapshot of patient's experiences with clinic services both at the stationary clinic and at the outreach campaigns.

\section{Conclusion}

The study findings that there was low knowledge of the purpose of cervical cancer screening or the effective treatment available for the disease among patients led to the development and dissemination of a photonovella to describe the process of cervical cancer screening at the campaigns. This pamphlet contributed to the educational component of the periodic campaigns conducted primarily in rural areas of Andean Peru to supplement the information received from the doctor or nurse-midwife during the Pap test. In addition, CerviCusco has recently expanded its cervical cancer prevention services to include the HPV vaccine at no cost to patients. In 2016, they are administering 20,000 doses of the vaccine to eligible patients. CerviCusco has demonstrated its capacity to provide screening outreach campaigns to populations who had not previously had access to liquid-based cytology services. CerviCusco reaches vulnerable populations in the rural highlands to help to reduce the coverage gap that exists in contemporary Peru.

\section{Acknowledgments}

This work was supported by the National Cancer Institute under Grant R03 CA173105. The content is solely the responsibility of the authors and does not necessarily represent the official views of the National Cancer Institute or the National Institutes of Health. We acknowledge the collaboration of the CerviCusco staff and the Peruvian Ministry of Health. We also acknowledge the research staff at Augusta University, CerviCusco, and Georgia Southern University who helped with the multi-institutional institutional review board approval process.

\section{Disclosure}

The authors report no conflicts of interest in this work.

\section{References}

1. HPV Information Centre. Human Papillomavirus and Related Cancers in Peru. Summary Report; 2016. Available from: http://www.hpvcentre. net/. Accessed June 7, 2016.

2. IARC. Globocan 2012: Estimated Cancer Incidence, Mortality and Prevalence Worldwide in 2012; 2012. Available from: http://globocan.iarc. fr/Pages/summary_table_site_sel.aspx. Accessed August 21, 2015.

3. Paz Soldan VA, Lee FH, Carcamo C, Holmes KK, Garnett GP, Garcia P. Who is getting Pap smears in urban Peru? Int J Epidemiol. 2008;37(4): $862-869$.
4. Winkler J, Bingham A, Coffey P, Handwerker WP. Women's participation in a cervical cancer screening program in northern Peru. Health Educ Res. 2008;23(1):10-24.

5. Paz-Soldan VA, Bayer AM, Nussbaum L, Cabrera L. Structural barriers to screening for and treatment of cervical cancer in Peru. Reprod Health Matters. 2012;20(40):49-58.

6. Bayer AM, Nussbaum L, Cabrera L, Paz-Soldan VA. Missed opportunities for health education on Pap smears in Peru. Health Educ Behav. 2011;38(2):198-209.

7. RINC/UNASUR. Informe preliminar reducido sobre la situación del control del cáncer de cuello uterino en 8 países de Latinoamérica. Network of National Cancer Institutes of the South American Union; 2012.

8. Soneji S, Fukui N. Socioeconomic determinants of cervical cancer screening in Latin America. Rev Panam Salud Publica. 2013;33(3): 174-182.

9. Park IU, Diaz ML, Bowey C, Magaril R, Ferris DG. Cervical cancer prevention in the developing world. J Low Genit Tract Dis. 2007;11(4):273.

10. Agurto I, Bishop A, Sanchez G, Betancourt Z, Robles S. Perceived barriers and benefits to cervical cancer screening in Latin America. Prev Med. 2004;39(1):91-98.

11. Bartolini RM, Winkler JL, Penny ME, LaMontagne DS. Parental acceptance of HPV vaccine in Peru: a decision framework. PLoS One. 2012;7(10):e48017.

12. Penny M, Bartolini R, Mosqueira NR, et al. Strategies to vaccinate against cancer of the cervix: feasibility of a school-based HPV vaccination program in Peru. Vaccine. 2011;29(31):5022-5030.

13. Bruni L, Diaz M, Barrionuevo-Rosas L, et al. Global estimates of human papillomavirus vaccination coverage by region and income level: a pooled analysis. Lancet Glob Health. 2016;4(7):e453-e463.

14. Patel H, Wilson E, Vizzotti C, Parston G, Prestt J, Darzi A. Argentina's successful implementation of a national human papillomavirus vaccination program. Health Aff (Millwood). 2016;35(2):301-308.

15. Ferris DG, Park I, Waxman A, et al. Initiating the American Society for Colposcopy and Cervical Pathology Humanitarian Program: Peru 2005. J Low Genit Tract Dis. 2006;10(1):58-62.

16. National Cancer Institute. Health Information National Trends Survey (HINTS); 2016. Available from: http://hints.cancer.gov/. Accessed April 7, 2016.

17. Luque JS, Tarasenko YN, Maupin JN, et al. Cultural beliefs and understandings of cervical cancer among Mexican immigrant women in Southeast Georgia. J Immigr Minor Health. 2014;17:713-721.

18. Deyo RA, Diehl AK, Hazuda H, Stern MP. A simple language-based acculturation scale for Mexican Americans: validation and application to health care research. Am J Public Health. 1985;75(1):51-55.

19. Robles SC, Ferreccio C, Tsu V, et al. Assessing participation of women in a cervical cancer screening program in Peru. Rev Panam Salud Publica. 2009;25(3):189-195.

20. Levinson KL, Abuelo C, Salmeron J, et al. The Peru Cervical Cancer Prevention Study (PERCAPS): the technology to make screening accessible. Gynecol Oncol. 2013;129(2):318-323.

21. Sankaranarayanan R. Screening for cancer in low- and middle-income countries. Ann Glob Health. 2014;80(5):412-417.

22. Bermedo-Carrasco S, Pena-Sanchez JN, Lepnurm R, Szafron M, Waldner C. Inequities in cervical cancer screening among Colombian women: a multilevel analysis of a nationwide survey. Cancer Epidemiol. 2015;39(2):229-236.

23. Stormo AR, Altamirano VC, Perez-Castells M, et al. Bolivian health providers' attitudes toward alternative technologies for cervical cancer prevention: a focus on visual inspection with acetic acid and cryotherapy. J Womens Health (Larchmt). 2012;21(8):801-808.

24. Ferris DG, Shapiro J, Fowler C, Cutler C, Waller J, Guevara Condorhuaman WS. The impact of accessible cervical cancer screening in Peru-The Dia del Mercado Project. J Low Genit Tract Dis. 2015; 19(3):229-233.

25. Levinson KL, Abuelo C, Chyung E, et al. The Peru cervical cancer prevention study (PERCAPS): community-based participatory research in Manchay, Peru. Int J Gynecol Cancer. 2013;23(1):141-147. 
26. Murillo R, Almonte M, Pereira A, et al. Cervical cancer screening programs in Latin America and the Caribbean. Vaccine. 2008;26(Suppl 11): L37-L48.

27. Camargo M, Soto-De Leon SC, Sanchez R, Perez-Prados A, Patarroyo ME, Patarroyo MA. Frequency of human papillomavirus infection, coinfection, and association with different risk factors in Colombia. Ann Epidemiol. 2011;21(3):204-213.

28. Ferris DG, Hupman C, Waller JL, Cudnik J, Watkins C. Assessment of the impact of cervical cancer prevention educational videos for Quechua- and Spanish-speaking Peruvian women. J Low Genit Tract Dis. 2009;13(4):244-251.

29. Ferris DG, Condorhuaman WS, Waller J, Lilienthal A. Impact of a video intervention for rural Peruvian women with cervical neoplasia before loop excisional procedures. J Low Genit Tract Dis. 2015;19: 229-233.
30. Luque JS. The faces on our educational materials: real stories behind the messages. J Cancer Educ. Epub 2016 Mar 15.

31. Tesla G. How a Team of IBM and BD Volunteers Are Helping a Local Clinic Save Lives in Peru's Andes Mountains; 2016. Available from: https://www.newglobalcitizen.com/global-pro-bono/team-ibm-bdvolunteers-helping-local-clinic-save-lives-perus-andes-mountains. Accessed September 1, 2016.

32. Han CS, Ferris DG, Waller J, Tharp P, Walter J, Allmond L. Comparison of knowledge and attitudes toward human papillomavirus, HPV vaccine, pap tests, and cervical cancer between US and Peruvian women. J Low Genit Tract Dis. 2012;16(2):121-126.

33. Lee FH, Paz-Soldan VA, Carcamo C, Garcia PJ. Knowledge and attitudes of adult Peruvian women vis-a-vis Human Papillomavirus (HPV), cervical cancer, and the HPV vaccine. J Low Genit Tract Dis. 2010;14(2):113-117.

\section{Publish your work in this journal}

Patient Preference and Adherence is an international, peer-reviewed, open access journal that focuses on the growing importance of patient preference and adherence throughout the therapeutic continuum. Patient satisfaction, acceptability, quality of life, compliance, persistence and their role in developing new therapeutic modalities and compounds to optimize clinical outcomes for existing disease states are major areas of interest for the journal. This journal has been accepted for indexing on PubMed Central. The manuscript management system is completely online and includes a very quick and fair peer-review system, which is all easy to use. Visit http://www dovepress.com/testimonials.php to read real quotes from published authors. 